\title{
A Giant Gastric Ulcer Caused by Mucormycosis Infection in a Patient with Renal Transplantation
}

\begin{abstract}
A 70-year-old man underwent cadaver renal transplantation. One month after transplantation, he had persistent lowgrade fever, cough, and progressive epigastric pain with intermittent tarry stool Panendoscopy revealed a giant gastric ulcer in the greater curvature of stomach (Figure 1 a), with dirty debris and a deep hemorrhagic base (Figure 1 b). Gastric biopsy confirmed a diagnosis of mucormycosis, with multiple nonseptate rightangled branched fungal hyphae on the hematoxylin-eosin stain (Figure 2). The silver stain showed characteristic vascular and epithelial invasion in the gastric mucosa (Figure 3). The fungus culture was negative. Amphotericin B infusion was started immediately after endoscopy. The patient had massive ulcer bleeding on the fifth day after endoscopy, and died ten days later without autopsy.
\end{abstract}

The endoscopic appearance of gastric mucormycosis may suggest the diagnosis, which is confirmed by a gastric biopsy $(1-3)$ demonstrating the typical characteristics of the condition. Culture of mucormycosis is usually negative (1). Amphotericin B infusion is an appropriate treatment $(1,2,4)$. However, a large ulcer such as this carries a high risk of massive ulcer bleeding, so that amphotericin B treatment is of limited efficacy in preventing a fatal outcome. Surgical interyention with gastric resection might be considered as an alternative in such cases.

B.-S. Sheu', P.-C. Lee ${ }^{2}$, H.-B. Yang ${ }^{3}$

'Dept. of Internal Medicine

${ }^{2}$ Dept. of General Surgery

${ }^{3}$ Dept. of Pathology,

National Cheng Kung

University Hospital, Tainan, Taiwan 

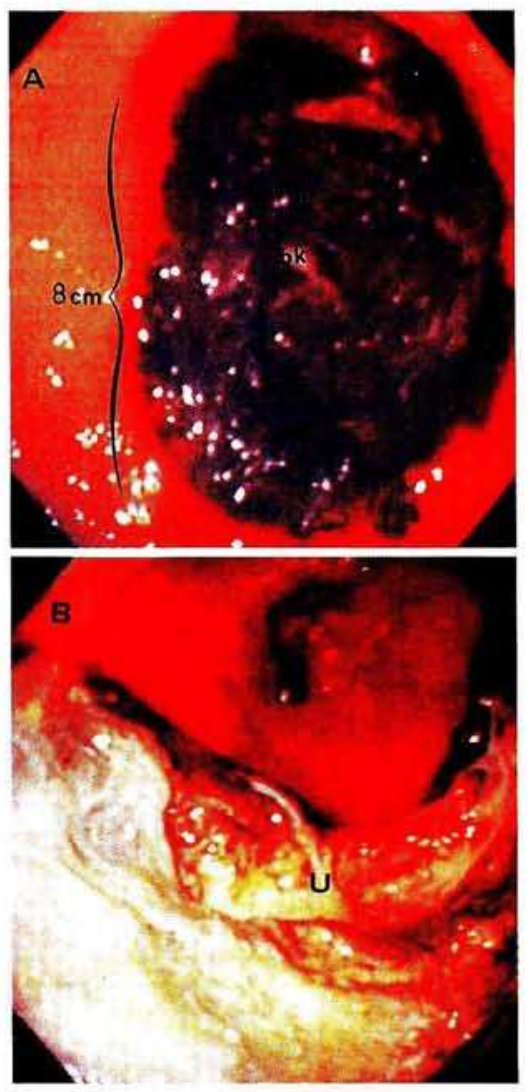

Figure 1A: The giant ulcer caused by the mucormycosis was over $8 \mathrm{~cm}$ in size (curved bracket). B The deep ulcer base (B) had a hemorrhagic edge $(U)$.

\section{References}

1. Brullet E, Andreu X, Elias J, Roig J, Cervantes M. Gastric mucormycosis in a patient with acquired immunodeficiency syndrome. Gastrointest Endosc 1993: 39: 106-7.

2. Winkler S, Susani S, Willinger B, et al. Gastric mucormycosis due to Rhizopus oryzae in a renal transplant recipient. J Clin Microbiol 1996; 34: 2585-7.

3. Ismail MH, Hodkinson HJ, Setzen G, Sofianos C, Hale MJ. Gastric mucormycosis. Trop Gastroenterol 1990; 11: $103-5$.

4. Parfrey NA. Improved diagnosis and prognosis of mucormycosis: a clinicopathological study of 33 cases. Medicine (Baltimore) 1986; 65: 113-23.

Corresponding Author

P.-C. Lee, M.D.

Dept. of General Surgery

National Cheng Kung

University Hospital

138 Sheng Li Road

Tainan, Taiwan

Fax: +886-6-2766676

E-mail: pochang@mail.ncku.edu.tw

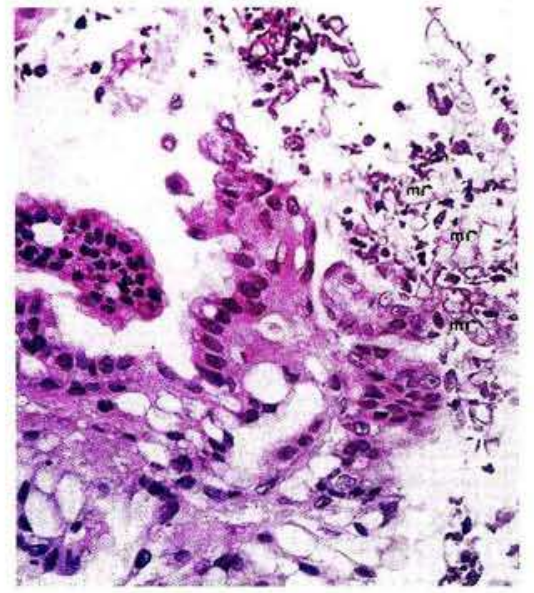

Figure 2: The gastric biopsy showed the typical nonseptate, right-angled ( $\mathrm{mr}$ ) branched hyphae of mucormycosis and inflammation of the gastric epithelium (hematoxylin-eosin, original magnification $\times 400$ )

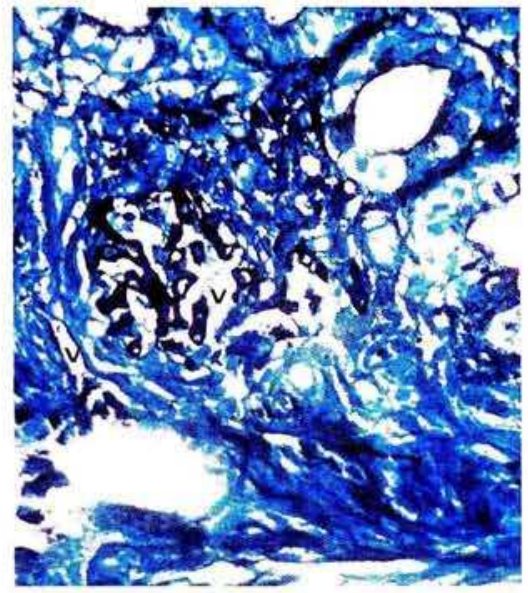

Figure 3: The silver stain (original magnification $\times 400$ ) further confirmed the diagnosis of mucormycosis, showing the characteristic vascular (V) and epithelial (E) invasion. The fungal organism is deeply stained (M). 